\title{
Avaliação comparativa do impacto na qualidade de vida, tempo cirúrgico e dor no pós operatório em mulheres portadoras de incontinência urinária de esforço submetidas à terapêutica cirúrgica pelas técnicas de "slings" pubovaginal e transobturador
}

Evaluation comparative of the impact on the quality of life, surgical time and postsurgical pain of women with stress urinary incontinence, who were submitted to surgical therapy by the slings techniques pubovaginal and transobturator

Autora: Danielle Cristiane Limonge Schwab

Orientadora: Profa. Dra. Aparecida Maria Pacetta

Co-orientadora: Silvia da Silva Carramão

Dissertação apresentada à Faculdade de Medicina da Universidade de Santo Amaro (UNISA) para obtenção do Título de Mestre em Saúde Materno-Infantil, em 24 de junho de 2005.

Objetivo: avaliar de forma comparativa o impacto na qualidade de vida de mulheres portadoras de incontinência urinária de esforço (IUE) submetidas à terapêutica cirúrgica pelas técnicas de "slings": pubovaginal e transobturador, tempo cirúrgico e dor no pós-operatório. Método: estudo experimental, prospectivo, casualizado para as técnicas, estudadas 39 mulheres portadoras de IUE, divididas em grupos grupos: GI (21 submetidas à "sling" pubovaginal com faixa sintética de colágeno suíno) e GII (18 submetidas à "sling" transobturador com faixa sintética de ácido poligalactina e policaprolactona). Resultados: aplicando a Análise de Variância de Friedman, aos valores atribuídos aos questionários de qualidade de vida (IQOL) pré e pós-cirúrgico, observou-se que ambas as técnicas mostraram-se eficientes no restabelecimento da continência urinária $(\mathrm{p}<0,001)$. Avaliando o tempo cirúrgico pelo teste de Mann-Whitney, houve diminuição do mesmo na técnica transobturadora em relação à pubovaginal $(\mathrm{p}<0,001)$. Analisando a variável dor pelo teste de Qui-quadrado ou teste Exato de Fisher, no pós-operatório dos grupos, observou-se melhora estatisticamente significante na via transobturadora; no pós-operatório imediato $(\mathrm{p}<0,001)$ e primeira semana de pós-operatório $(\mathrm{p}<0,031)$. Conclusão: a técnica transobturadora é uma nova via simples, eficaz e segura para a correção da IUE.

PALAVRAS-CHAVE: Incontinência urinária; Qualidade de vida; "Sling" transobturador 